\title{
Evidence of Insecticide Resistance to Pyrethroids and Bendiocarb in Anopheles funestus from Tsararano, Marovoay District, Madagascar
}

\author{
Tsiriniaina Rakotondranaivo, ${ }^{1,2}$ Solohery Fanomezana Randriamanarivo, ${ }^{1}$ \\ Mihajarilala Rakotoniaina Tanjona, ${ }^{1}$ Inès Vigan-Womas, ${ }^{3}$ \\ Milijaona Randrianarivelojosia, ${ }^{4,5}$ and Mamadou Ousmane Ndiath (iD ${ }^{1}$ \\ ${ }^{1}$ Institut Pasteur de Madagascar, G4 Malaria Group, Ambatofotsikely, Antananarivo 101, Madagascar \\ ${ }^{2}$ Ecole Doctorale Génie du Vivant et Modélisation, Université de Mahajanga, Mahajanga 401, Madagascar \\ ${ }^{3}$ Institut Pasteur de Madagascar, Unité d'Immunologie des Maladies Infectieuses, Ambatofotsikely, Antananarivo 101, Madagascar \\ ${ }^{4}$ Institut Pasteur de Madagascar, Unité de Recherche sur le Paludisme, Ambatofotsikely, Antananarivo 101, Madagascar \\ ${ }^{5}$ Faculté des Sciences, Université de Toliara, Toliara 601, Madagascar \\ Correspondence should be addressed to Mamadou Ousmane Ndiath; ousmane.ndiath@gmail.com
}

Received 5 March 2018; Revised 17 May 2018; Accepted 20 September 2018; Published 8 October 2018

Guest Editor: Ademir J. Martins

Copyright ( 2018 Tsiriniaina Rakotondranaivo et al. This is an open access article distributed under the Creative Commons Attribution License, which permits unrestricted use, distribution, and reproduction in any medium, provided the original work is properly cited.

\begin{abstract}
Introduction. In Madagascar, malaria control relies on the countrywide use of long lasting insecticide treated bed nets (LLINs) and on indoor residual spraying (IRS) in the central highland area as well as a small area on the eastern coast. We tested insecticide resistance mechanisms of Anopheles funestus from Tsararano, a malaria endemic village in the coastal health district of Marovoay. Methods. Insecticide susceptibility bioassays were done in July 2017 on first-generation Anopheles funestus (F1) to assess (i) the susceptibility to permethrin $(0.05 \%)$, deltamethrin (0.05\%), DDT (4\%), malathion (5\%), fenitrothion (1\%), and bendiocarb (0.1\%); (ii) the effect of preexposure to the piperonyl butoxide (PBO) synergist; and (iii) the enzymatic activities of cytochrome P450, esterases, and glutathione S-transferases (GST). Results. Our results demonstrated that An. funestus was phenotypically resistant to pyrethroids and bendiocarb, with a mortality rate (MR) of 33.6\% (95\%CI: $24.5-43.7 \%)$ and 86\% (95\%CI: 77.6-92.1\%), respectively. In contrast, An. funestus were $100 \%$ susceptible to DDT and organophosphates (malathion and fenitrothion). Preexposure of $A n$. funestus to PBO synergist significantly restored the susceptibility to bendiocarb (MR=100\%) and increased the MR in the pyrethroid group, from $96 \%$ (95\%CI: 90.0-98.9\%) to $100 \%$ for deltamethrin and permethrin, respectively $\left(\chi^{2}=43, d f=3, P<0.0001\right)$. Enzymatic activities of cytochrome P450 and $\alpha$-esterases were significantly elevated among An. funestus compared with the IPM reference strain (Mann-Whitney $U=30, P<0.0001 ; U=145.5, P<0.0001$, respectively). No significant differences of $\beta$-esterases activities compared to the IPM reference strain were observed (Mann-Whitney $U=392.5, P=0.08$ ). Conclusion. In Tsararano, despite the absence of an IRS programme, there is evidence of high levels of insecticide resistance to pyrethroids and bendiocarb in An. funestus. Biochemical data indicated that a metabolic resistance mechanism through the cytochrome $\mathrm{P} 450$ genes is operating in the An. funestus population.
\end{abstract}

\section{Background}

Malaria remains a major public health burden on the island of Madagascar. Today, despite the fight against malaria which is mainly based on the use of rapid diagnostic test (RDT), the administration of artemisinin-based combination therapies (ACTs) as first-line treatment, and the implementation of vector control measures, malaria remains the third leading cause of morbidity and mortality in Madagascar, behind respiratory infections and diarrhea [1]. In 2015, malaria morbidity was $10.1 \%$ for all ages and $18.6 \%$ for children under 5 years of age. The malaria-related mortality ranged from $12.3 \%$ to $25.7 \%$ and incidence from $3.1 \%$ to $6.7 \%$ among those under 5 years of age $[2,3]$. Thus, malaria still hampers 
the prosperity and economic development of the country as the economic cost of malaria is estimated at more than 50 million USD per year [4]. Consequently, in Madagascar, these malaria control measures must be increased to achieve the global malaria action plan (GMAP) [5].

Madagascar deployed traditional vector control methods as early as $1949[6,7]$. The global malaria eradication campaign in the 1950s and 1960, which was based on the large-scale use of dichlorodiphenyltrichloroethane (DDT), was successful in Madagascar and there was a significant drop in national malaria transmission from 1959 until the early 1970s [8]. Unfortunately, these efforts were not sustained and recurring malaria outbreaks were observed in the central highlands in the 1980s $[9,10]$. The upsurge of the disease was due to several factors such as the abandonment of indoor residual spraying (IRS), insufficient funding for malaria control, the erosion of the public health system, and the poor knowledge of the malaria vector biology (including that of Anopheles funestus, the main vector involved in the malaria epidemics in the central highlands) as well as the Anopheles resistance to insecticides [11-13].

The diversity of Madagascar's ecosystems with 5 epidemiological zones, several vectors, 2 major parasites $(P$. falciparum and $P$. vivax), and the changes they experience as a result of human activities (e.g. the use of insecticides for agriculture and vector control), among others, renders the malaria vector system extremely complex and constantly evolving. Today, it is widely accepted that vector control strategies will only be effective in the long term if there is a thorough knowledge of vector biology and, particularly, the status of insecticide resistance $[14,15]$. The failure to take into account insecticide susceptibility of the potential vectors involved in malaria transmission has contributed to failures in malaria control throughout Africa [5].

The most effective way to prevent malaria transmission is to avoid human-vector contact, hence the importance of vector control [5]. However, in many countries where vector control strategies, including IRS and long lasting insecticide treated bed nets (LLINs), have been implemented on a large scale, profound vector changes have been observed. These range from behavioral changes to insecticide resistance, which greatly undermine the success of current vector control $[16,17]$. This situation is all the more compelling when considering that Anopheles exhibits a strong resistance to pyrethroids [5], the only insecticide recommended and approved by the WHO for bed net impregnation [18]. In several countries, resistance to pyrethroids has been reported in major malaria vectors including An. gambiae s.l. and An. funestus $[19,20]$. This has become an almost universal problem and may seriously impair the progress noticed in fighting malaria [5].

In Madagascar, IRS using DDT has been implemented since the 1950s whereas LLINs were widely distributed in the last decade $[9,21]$. Malaria control still relies on the use of LLINs throughout the country and on IRS in the central highland area and in a small area on the eastern coast [22].

In malaria endemic regions, mosquito resistance can increase rapidly following the implementation of vector control [17]. Therefore, it is crucial to monitor the resistance

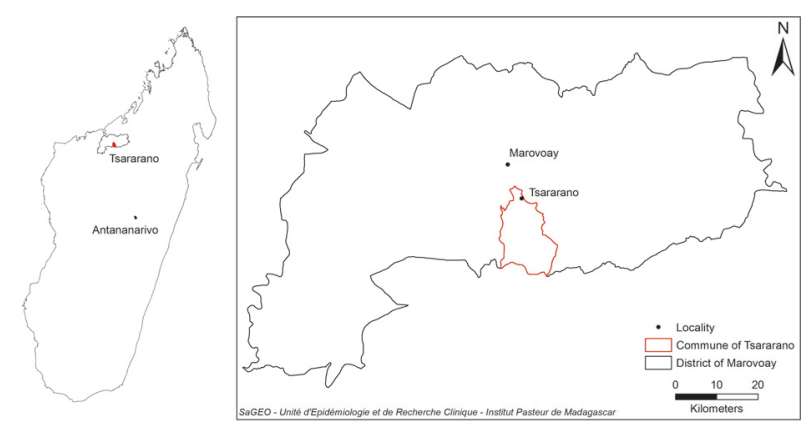

Figure 1: Map of Marovoay district (Madagascar) showing study Tsararano area.

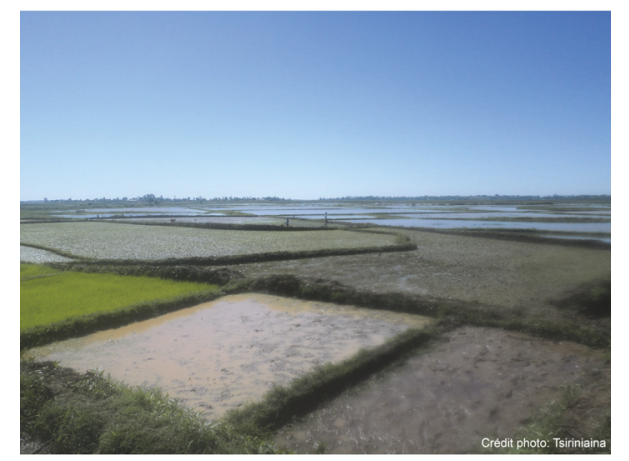

FIGURE 2: Photograph showing the vast rice fields at Tsararano Marovoay constituting potential larval breeding site for Anopheles funestus.

to insecticides, particularly in countries where the policy of universal bed net coverage is applied. Recent studies demonstrated the occurrence of the resistance to pyrethroids and DDT in An. arabiensis population in several districts of Madagascar [23]. However, little information is available on the status and insecticide resistance mechanisms in $A n$. funestus, one of the main malaria vectors [24]. In this paper, we report the insecticide susceptibility in wild An. funestus population from Tsararano and the involved resistance mechanisms.

\section{Methods}

2.1. Study Site. This study was carried out in the village of Tsararano (S $16^{\circ} 10^{\prime} 42.4^{\prime \prime}$, E $\left.046^{\circ} 40^{\prime} 13.6^{\prime \prime}\right)$, located in the district of Marovoay, in the Boeny region, approximately $540 \mathrm{~km}$ northwest of Antananarivo, the capital city of Madagascar (Figure 1). The village has about 1,200 inhabitants. It has a tropical climate and rainfall occurs from October to April. The average annual temperature is $29^{\circ} \mathrm{C}$ and the average rainfall is $1,500 \mathrm{~mm}$. Tsararano is situated on the marshy bank of a permanent stream river, the Betsiboka. In this second largest rice granary region of Madagascar, agriculture is the main activity (Figure 2). Anopheles larval sites are present all year round and malaria is endemic. No IRS has been implemented in the district since the 1950s; however, LLINs were distributed for the fifth round in December 2015. 
2.2. Mosquito Collection. Blood fed female mosquitoes were collected in the morning between 6 and 10 am from ten (10) households during the dry season in July 2017, using a mouth aspirator and transferred at the insectary of CSB2 Andriba platform. After morphological identification [25], one hundred and eighty-six (186) blood fed An. funestus were individually transferred into a tube (Eppendorf ${ }^{\circledR}$ ) for forced oviposition according to the method described by Nepomichene et al. [26]. In brief, female mosquitoes were maintained at $28^{\circ} \mathrm{C}$ in $75-80 \%$ relative humidity with free access to $10 \%$ sucrose until they became fully gravid (about four days) and individually placed inside a tube with a filter paper placed at the bottom. Mosquitoes were introduced inside the tube one by one from within the cage. Oviposited eggs from each female were counted and pooled and were subsequently placed into a rearing pan containing wellwater. Pooled larvae of different stages L1, L2, L3, and L4 were fed daily with Tetramin ${ }^{\mathrm{TM}}$ baby fish food. Pupae were collected and placed in 2-L plastic buckets, which were covered with mosquito gauze with a cotton sleeve for introducing $10 \%$ glucose on filter paper and allowed to emerge locally. Unfed 2-5-day females on first-generation An. funestus (F1) were used for insecticide susceptibility bioassay.

\subsection{Susceptibility Assays. Insecticide susceptibility bioas-} says, following WHO protocol [27], were done on F1 An. funestus generation at the insectary of Andriba platform. Six insecticides of technical grade quality were tested: two pyrethroids (permethrin $0.75 \%$, deltamethrin $0.05 \%$ ), two organophosphates (malathion 5\%, fenitrothion 1\%), one organochlorine (DDT 4\%), and one carbamate (bendiocarb $0.1 \%)$. Impregnated papers were obtained from the $\mathrm{WHO}$ reference center (Vector Control Research Unit, University Sains Malaysia, Penang, Malaysia). Tests were performed with batches of 25 unfed females aged 2-5 days, fourfold with each insecticide. Mosquitoes were exposed for 1 hour at $28^{\circ} \mathrm{C}$ and $80 \%$ relative humidity. After exposure, mosquitoes were kept in observation tubes and supplied with a $10 \%$ sugar solution. The number of knockdown (KD) mosquitoes was recorded at 10, 15, 20, 30, 40, 50, and $60 \mathrm{~min}$. Mortality rate (MR) was recorded after 24 hours. Two batches of 25 females exposed to untreated papers were used as negative control. All batches of insecticide paper used were pretested on the strain of An. arabiensis from the Institut Pasteur de Madagascar known to be susceptible to all insecticides [28].

2.4. PBO Synergist Assays. In the event of confirmed insecticides resistance, additional tests were conducted by using the synergist piperonyl butoxide (PBO), an inhibitor of oxidases. Three combinations were used: $\mathrm{PBO}+$ deltamethrin, $\mathrm{PBO}+$ permethrin, and $\mathrm{PBO}+$ bendiocarb. For each combination, two exposed batches and one negative control batch exposed only to PBO were used. Each batch was constituted by 25 unfed female mosquitoes aged 2-5 days old. Mosquitoes were first exposed for one hour to $4 \% \mathrm{PBO}$ and second to impregnated papers. KD mosquitoes and mortality rate were recorded as described above.
2.5. Biochemical Enzyme Assays. A subset of F1 An. funestus generation (nonexposed to insecticide) stored at $-80^{\circ} \mathrm{C}$ until use was used for biochemical enzyme assays. Enzymatic activities of cytochrome P450, esterases ( $\alpha$ and $\beta$ ), and glutathione S- transferases (GST) were measured according the protocol described by Brogdon et al. [29] and by Hemingway et al. [30], and slightly modified by Sangba et al. [31]. Absorbance was measured using a spectrophotometer type "Multiskan GO and SkanIt Software/Serial number 151005171" (https://www.thermofisher.com/us/en/home/life-science/lab-plasticware-supplies/lab-plasticware-supplies-learning-center/lab-plasticware-supplies-resource-library/skanItsoftware-protocols-multiskan-go.html). In the absence of An. funestus susceptible strain, the An. arabiensis strain from the Institut Pasteur de Madagascar known to be susceptible to all insecticides was used as control [28]. All experiments were conducted at the laboratory of the Institut Pasteur de Madagascar.

2.6. Species Molecular Identification. DNA was extracted from all F0 individual mosquitoes by DNAzol (Invitrogen, CA, USA) according to the manufacturer's recommendations. Anopheles mosquitoes were identified by the PCR using the method described by Santolamazza et al. for An. gambiae, and the technique described by Wilkins et al. for An. funestus [32]. The PCR for An. gambiae s.l. discrimination is based on the amplification of a ribosomal DNA fragment smaller amplified by PCR by using the following primers: IGS441 (F) [TGG TCT GGG GAC CAC GTC GAC ACA GG], IGS783 (R) [CGT TTC TCA CAT CAA GAC AAT CAA GTC], while the PCR for An. funestus discrimination is based on species-specific single nucleotide polymorphism (SNPs) in the second internal transcribed spacer region (ITS2) by using seven primers: UV (F,) [CCG ATG CAC ACA TTC TTG AGT GCC TA], FUN (R) [CTC GGG CAT CGA TGG GTT AAT CAT G], VAN (R) [AAC TCT GTC GAC TTG GTA GCC GAA C], RIV (R) [AAT CAG GGT CGA ACG GCT TGC CG], PAR (R) [GCC CTG CGG TCC CAA GCT AGA TT], RIVLIKE (R) [CTC CCG TGG AGT GGG GGA TC], LEES (R) [GAC GGC ATC ATG GCG AGC AGC]. All primers were provided by the Center for Diseases Control (CDC) and the experiments were done at the laboratory of Institut Pasteur de Madagascar.

2.7. Data Analysis. For each insecticide, the percentage of KD and MR was determined. Since mortality in negative controls was always under 5\%, no adjustment was performed for treated batches. The WHO 2017 criteria were used to evaluate the status of insecticide resistance (i.e., 98-100\% mortality indicates susceptibility and $<98 \%$ mortality indicates that further investigation is required to confirm resistance) [27]. Fifty and ninety-five percent $\mathrm{KD}$ times $\left(\mathrm{KDT}_{50}\right.$ and $\mathrm{KDT}_{95}$, respectively) were computed with probit regression models. Mortality rates expressed in (\%) and 95\% confidence interval were compared using Fisher exact test. The enzymatic activities of wild An. funestus were compared with that of the An. arabiensis strain from the Institut Pasteur de Madagascar known to be susceptible to all insecticides by Mann-Whitney test. Statistical analyses were performed using GraphPad $^{\circledR}$ 
TABLE 1: Anophelines collected in Tsararano by indoor aspirator and molecular identification by PCR and circumsporozoite protein rate (CSP). Mosquitoes were captured in July 2017 during the dry season in ten (10) households at early morning between 6 and 10 am.

\begin{tabular}{|c|c|c|c|}
\hline \multicolumn{4}{|c|}{ Morphological identification } \\
\hline Methods & Anopheles funestus s.l. & \multicolumn{2}{|c|}{ Anopheles gambiae s.l. } \\
\hline Indoor aspirator mosquito catch & 254 & \multicolumn{2}{|c|}{57} \\
\hline \multicolumn{4}{|c|}{ molecular identification } \\
\hline \multirow[t]{2}{*}{ PCR species } & Anopheles funestus s.s. & Anopheles arabiensis & Anopheles gambiae \\
\hline & 254 & 55 & 2 \\
\hline \multicolumn{4}{|c|}{ Plasmodium falciparum circumsporozoite protein rate } \\
\hline Number of Positive ELISA & 3 & 1 & 0 \\
\hline CSP rate $(\%)$ & $1.2 \%$ & $1.8 \%$ & 0 \\
\hline
\end{tabular}

TABLE 2: Mortality rate (MR) 24 hours after exposition obtained after WHO bioassay first-generation An. funestus population (F1) from Tsararano, Marovoay, and knockdown time $50\left(\mathrm{KDT}_{50}\right)$ and knockdown time $95\left(\mathrm{KDT}_{95}\right)$. Mortality rate represents mean with $95 \%$ confidence intervals (CI). na: not applicable.

\begin{tabular}{|c|c|c|c|c|c|c|}
\hline & \multicolumn{2}{|c|}{ Mortality rate (\%) } & \multicolumn{2}{|c|}{ Knockdown time 50} & \multicolumn{2}{|c|}{ Knockdown time 95} \\
\hline & $\operatorname{MR}(\mathbf{n})$ & 95\%CI & $\mathrm{KDT}_{50}$ & 95\%CI & $\mathrm{KDT}_{95}$ & $95 \% \mathrm{CI}$ \\
\hline Permethrin $0.75 \%$ & $33.6(101)$ & {$[24.5-43.7]$} & 73.79 & {$[70.4-77.1]$} & na & - \\
\hline Deltamethrin $0.05 \%$ & $39(100)$ & {$[29.4-49.2]$} & 72.5 & {$[68.1-76.9]$} & na & - \\
\hline Bendiocarb 0.1\% & $86(100)$ & {$[77.6-92.1]$} & 38.1 & {$[36.4-39.7]$} & na & - \\
\hline DDT 4\% & $99(101)$ & - & 34.4 & {$[32.4-36.4]$} & na & - \\
\hline Malathion 5\% & $100(99)$ & - & 28.9 & {$[25.6-32.2]$} & 39.0 & {$[36.9-42.0]$} \\
\hline Fenitrothion 1\% & $100(102)$ & - & 26.1 & {$[22.7-29.6]$} & 48.7 & {$[44.7-52.7]$} \\
\hline
\end{tabular}

Prism software v5.0 (www.graphpad.com). A $P$ value of 0.05 or less was considered as significant.

\section{Results}

3.1. Wild Anopheles Collections. In July 2017, a total of 311 malaria vectors were collected from 10 households in Tsararano. Among those, 254 (81.7\%) were An. funestus and 57 (18.3\%) An. gambiae s.l. (Table 1). Most of these mosquitoes were blood-fed. From the 186 oviposition tubes set up with individual gravid An. funestus females, 175 produced egg batches. The total number of eggs collected was 12,011, averaging 64.57 eggs per female.

3.2. Molecular Identifications and Infection Rates. All F0 An. funestus group mosquitoes $(\mathrm{n}=254)$ tested by PCR showed that they all belonged to An. funestus s.s. Of the $57 A n$. gambiae s.l. tested, 96.5\% $(\mathrm{n}=55)$ were identified as $A n$. arabiensis and $3.5 \%(\mathrm{n}=2)$ as An. gambiae (formerly S molecular form) (Table 1).

The Plasmodium falciparum circumsporozoite protein (CSP) rate was determined by ELISA-CSP. Of the 311 Anopheles specimens tested by ELISA-CSP, 4 were positive for the CSP antigen, resulting in a mean CSP rate of $1.3 \%(95 \% \mathrm{CI}$ : $0.35-3.2 \%$ ) with $1.2 \%$ and $1.8 \%$ for An. funestus and $A n$. gambiae s.l., respectively $\left(\chi^{2}=0.12, d f=1, P=0.7\right)$.

3.3. Susceptibility of Anopheles funestus to Insecticides. Table 2 summarizes the MR of An. funestus population induced by all insecticides. Following $24 \mathrm{~h}$ observation, MRs indicated high resistance to pyrethroids and bendiocarb (Figure 3 and Table 2). The mean MR induced by permethrin and deltamethrin in the wild An. funestus population was $33.6 \%$ (95\%CI: 24.5-43.7\%) and 39\% (95\%CI: 29.4-49.2\%), respectively. No significant difference of the MR was observed in the pyrethroid group $(\chi 2=0.61, d f=1, P=0.4)$. The mean MR induced by bendiocarb in the wild An. funestus population was $86 \%$ (95\% CI: $77.1-92.1 \%)$.

An. funestus was $100 \%$ susceptible to DDT and organophosphate (malathion and fenitrothion) (Figure 3 and Table 2). Furthermore, $100 \%$ of mosquitoes were knockdown after 60 min exposure to malathion, fenitrothion, and DDT with a short $\mathrm{KDT}_{50}$ time $<28.9 \mathrm{~min}$ (95\%CI: 25.7-32.2) (Table 2). For pyrethroids the $\mathrm{KDT}_{50}$ was much longer (higher than $72 \mathrm{~min}$ ) (Table 2). Meanwhile, a low $\mathrm{KDT}_{50}$ was observed after bendiocarb exposure despite the resistance (Table 2).

3.4. Effects of Synergist PBO on Anopheles funestus Resistant to Pyrethroids and Bendiocarb. For both pyrethroids and bendiocarb, preexposure to PBO synergist significantly increased the MR (Figure 3). The mean MR of deltamethrin increased from 39\% (95\%CI: $29.4-49.2 \%$ ) before PBO exposure to $96 \%$ (95\%CI: 90-98.9\%) after PBO exposure. The MR induced by permethrin increased from $33.6 \%$ before $\mathrm{PBO}$ exposure to $100 \%$ after $\mathrm{PBO}$ exposure. A significant difference of MR was observed in pyrethroid group before and after $\mathrm{PBO}$ exposure $\left(\chi^{2}=43, d f=3, P<0.0001\right)$. Concurrently, a full susceptibility was observed to bendiocarb after $\mathrm{PBO}$ exposure $(\mathrm{MR}=100 \%)$ (Figure 3).

3.5. Biochemical Enzymatic Activities of Cytochrome P450, Esterases, and GST. Biochemical data of enzymatic activities 


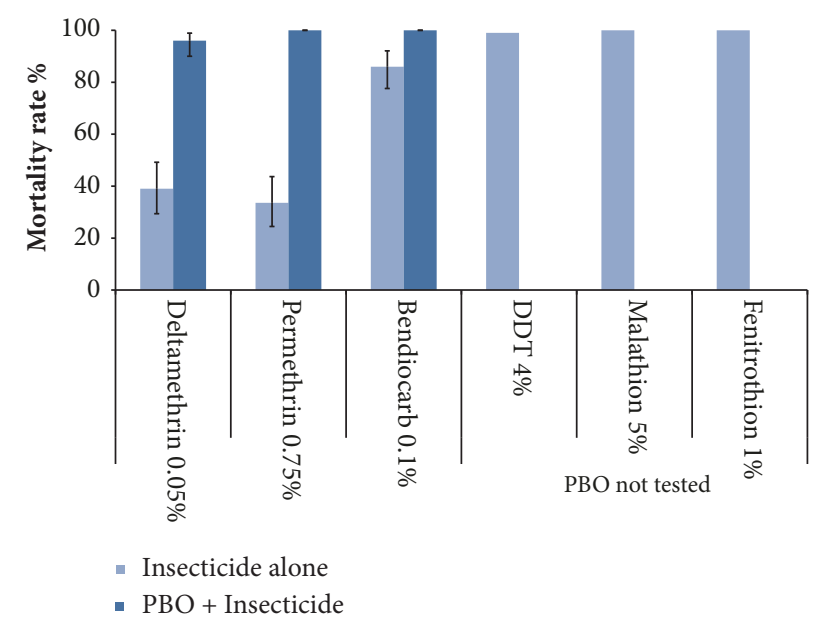

FIgURE 3: Insecticide bioassay mortality rate in wild Anopheles funestus first generation (F1) from Tsararano, Marovoay (Madagascar), 24 hours after exposure to permethrin (0.75\%), deltamethrin $(0.05 \%)$, DDT $(4 \%)$, malathion (5\%), fenitrothion (1\%), and bendiocarb $(0.1 \%)$ and effect of piperonyl butoxide $(\mathrm{PBO})$. The data represent medians.

of cytochrome P450, GST, and esterases ( $\alpha$ and $\beta$ ) compared to the IPM susceptible strain are shown in Figure 4. High level enzymatic activities of cytochrome P450 compared to the susceptible IPM reference strain were observed (MannWhitney $U=30, Z=-6.51, P<0.0001$ ) (Figure $4(\mathrm{a})$ ). In contrast, the GST activity was significantly lower compared to the IPM reference strain (Mann-Whitney $U=195.5, Z=-4.33$, $P<0.0001$ ) (Figure 4(b)) suggesting the no implication of GST in the insecticide resistance of An. funestus from Tsararano.

Meanwhile, a significant increased $\alpha$-esterase activity was observed in the An. funestus population compared to the IPM susceptible strain (Mann-Whitney $U=145.5, Z=-4.99, P$ $<0.0001$ ) (Figure $4(\mathrm{c})$ ). In contrast, no significant $\beta$-esterase activity was observed in the An. funestus populations from Tsararano compared to the IPM strain (Mann-Whitney $U=$ 392.5, $Z=-1.87, P=0.08$ ) (Figure $4(\mathrm{~d})$ ).

\section{Discussion}

This study, conducted in Tsararano, reports the levels of insecticide susceptibility of An. funestus, a main malaria vector in Madagascar, to the different families of insecticides conventionally used in vector control in the country.

Our study showed that An. funestus is the only species of the funestus group present in the study area with a moderate index circumsporozoite rate, which was considerably smaller than that obtained by Marrama et al. [13], and Fontenille et al. [11] in other parts of Madagascar. However, in many subSaharan Africa regions, entomological inoculation rate (EIR) of An. funestus surpasses that of An. gambiae, indicating their major role in malaria transmission throughout the continent $[17,33]$.

The study provides substantial knowledge on the resistance status of the wild An. funestus population. The results of insecticide bioassay trials showed a high biological level of resistance to pyrethroid groups (deltamethrin and permethrin) and bendiocarb. Similar resistance has been observed in southern east Africa, particularly in Mozambique [34], but in Madagascar, little information on An. funestus is available, in contrast to An. gambiae [23, 24]. Furthermore, investigations on the biological insecticide susceptibility performed in sentinel sites of Madagascar in 2015 reported a resistance to pyrethroids and bendiocarb in 20/37 and 8/24 sites, respectively, for An. gambiae. Meanwhile, resistance to the pyrethroid group and bendiocarb was observed only in $1 / 5$ and $1 / 3$ sites, respectively, for An. funestus, including in the district of Marovoay [24]. However, previous studies conducted in the Central Island of Madagascar by Rakotondraibe et al [35] and Ratovonjato et al [36] showed a full susceptibility to pyrethroids and DDT in wild blood feed and semigravid An. funestus population (F0) in contrast to our study where F1 progeny was used according to the WHO protocol [27]. This susceptibility difference may be due by several factors including the intensive uses of pesticides in Tsararano rice fields before 1950s [37] and also the age and physiological status of mosquitoes (i.e., blood fed and/or semigravid). For insistence, it has been demonstrated that older mosquitoes are sometimes less resistant to insecticides, especially when resistance is conferred by the presence of a detoxifying enzyme, the activity of which may decline with age [38].

On the other hand, previous studies have shown that the occurrence of the Anopheles resistance to insecticides is partly explained by the use of pesticides in agricultural activities and vector control to combat malaria [39, 40]. Given that Marovoay is the second largest rice granary in Madagascar, Tsararano is surrounded by rice fields where insecticides are used for pest control for an extended period of time throughout the year. Up to today, no IRS has been performed in Tsararano as part of the national vector control strategy. We hypothesize that the increased resistance of An. funestus to pyrethroids and bendiocarb observed in Tsararano might be due to the use of pesticides in agriculture. Nevertheless, the causes of such widespread resistance remain unknown. However, we believe LLINs would have a limited effect in spreading resistance in this region, as described elsewhere [41].

The long-term efficacy of LLINs in reducing malaria morbidity has recently been questioned in western Africa [17]. In Madagascar, 2,761,550 insecticide-treated nets were distributed for free to the population in 2016 [42]. Nevertheless, a full susceptibility to organophosphates has been observed in An. funestus population; therefore, these insecticides should be used in priority if IRS should be performed in this area.

The presence or absence of $k d r$ mutation in An. funestus population from Tsararano was not investigated. Several studies have shown the absence of this mutation in $A n$. funestus populations [41, 43] unlike in An. gambiae, where the widespread distribution of $k d r-w$ and $k d r-e$ has been observed in many African regions [5, 44, 45]. In addition, to our knowledge, no $k d r$ mutation was observed in An. gambiae populations from Madagascar [23].

Preexposure to $\mathrm{PBO}$ restored the full susceptibility to pyrethroids and bendiocarb. Similar observations have been 


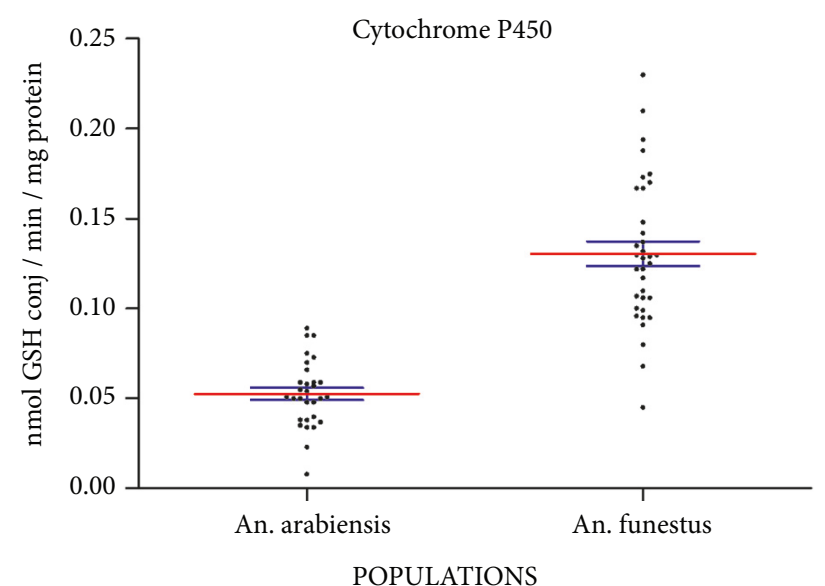

(a)

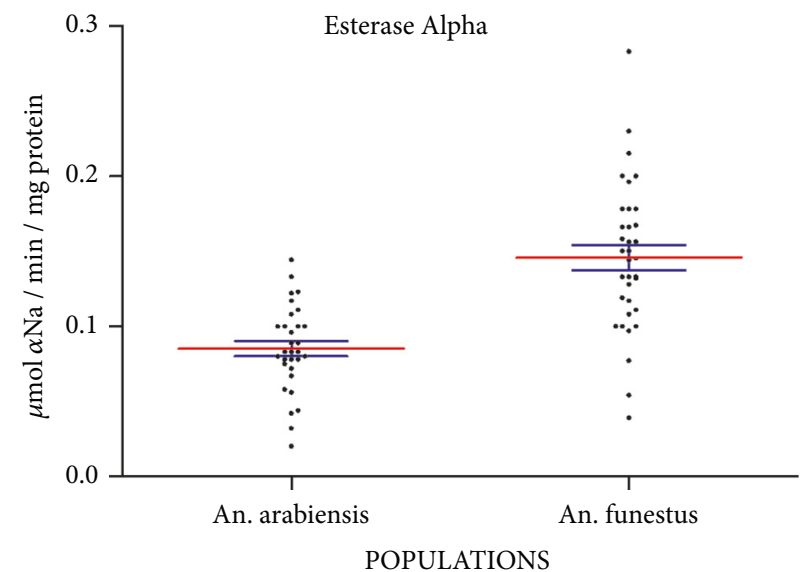

(c)

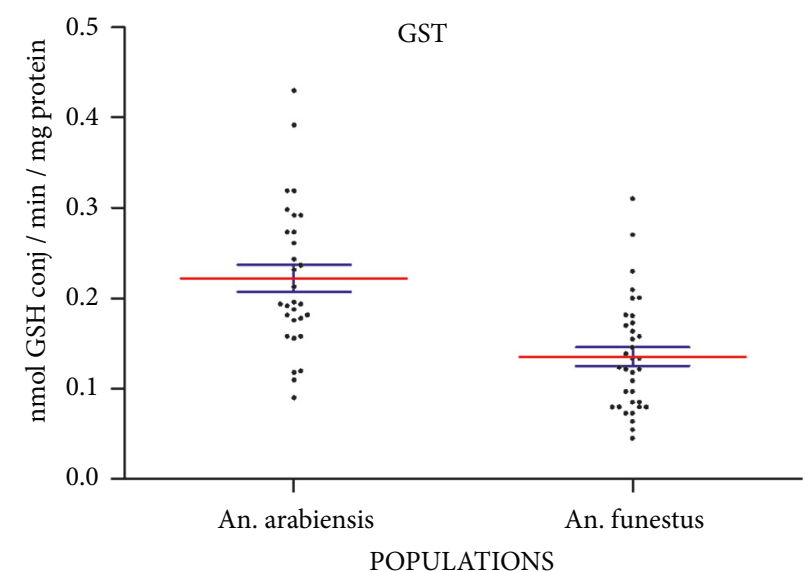

(b)

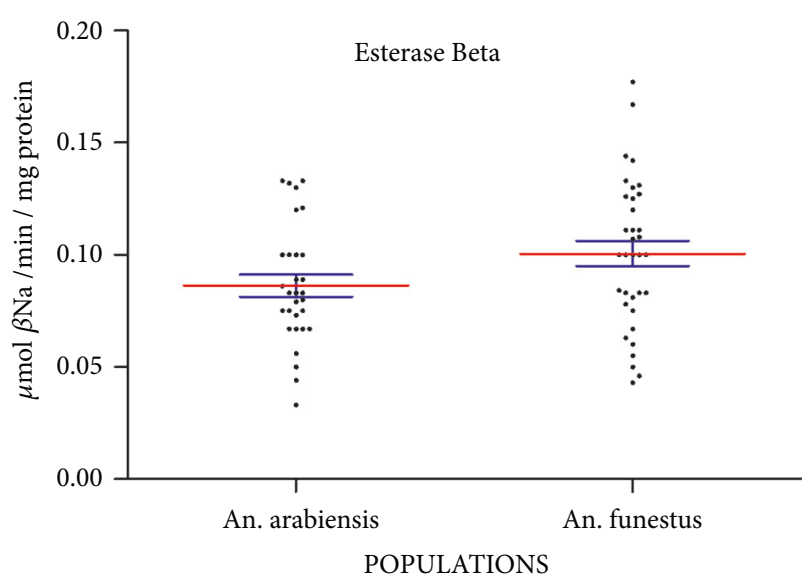

(d)

FIgURE 4: Detoxifying enzyme activities (enzymatic activity per mg of protein) of wild Anopheles funestus first generation (F1) from Tsararano, Madagascar, in comparison with Anopheles arabiensis Institut Pasteur of Madagascar susceptible strain. (a) Cytochrome P450 activities (MFO). (b) Glutathione S-transferases activities (GST). (c) Alpha esterase activities. (d) Beta esterase activities. Red lines represent means with $95 \%$ confidence intervals (blue lines).

made in Mozambique [34, 46]. Biochemical enzymatic activity data showed that cytochrome P450 activity was significantly higher in An. funestus population than the IPM strain indicating that the pyrethroid resistance in Tsararano is driven by the cytochrome $\mathrm{P} 450$ genes, as previously observed in Mozambique and in the Central African Republic $[34,46]$. In addition, we hypothesize that overexpression of cytochrome P450s also confers a cross-resistance to carbamates. This situation is particularly likely if the pyrethroid resistance results from a change in a P450 regulatory region, rather than a mutation in a single $\mathrm{P} 450$ structural gene, which could produce cross-resistance to several insecticide classes including carbamates, as previously observed in Mozambique [34]. Interestingly, previous studies revealed that pyrethroid resistance in An. funestus population is mainly driven by key cytochrome P450s genes such as CYP6P9a, CYP6P9b, and CYP6M7 [47, 48]. However, the gene expression pattern of the detoxification linked to this cross-resistance is not well understood.

Furthermore, a significant overexpression activity of $\alpha$-esterase was observed in An. funestus from Tsararano.
However, no difference in $\beta$-esterase activity was observed. In contrast, GST activity was significantly lower. This confirms the susceptibility of An. funestus from Tsararano to DDT. In fact, the evidence of resistance to the two major classes of insecticides (pyrethroids and bendiocarb) in the An. funestus population from Tsararano may constitute a potential threat to the success of the malaria vector control programme in this region. In addition, given the wide distribution of An. funestus in Madagascar, further investigation in other districts through the exploration of resistance profile is needed. This will provide information to elaborate adequate vector resistance management.

\section{Conclusions}

Our study showed evidence of resistance to pyrethroids and bendiocarb in an An. funestus population. Enzymatic activities data indicated implication of cytochrome P450 genes in the resistance of An. funestus with a suspected cross-resistance between pyrethroids and carbamate. The coexistence of this cross-resistance in An. funestus, where 
confirmed, constitutes a serious concern for the future success of malaria control programme.

\section{Data Availability}

The mortality rate data used to support the findings of this study are included within the article and the enzymatic activities data used to support the findings of this study are available from the corresponding author upon request.

\section{Ethical Approval}

This study is a part of G4 project approved by the national ethics committee in Madagascar (authorization no 122MSANP/CE-2015).

\section{Disclosure}

The funders had no role in study design, data collocation or analysis, decision to publish, or preparation of the manuscript.

\section{Conflicts of Interest}

The authors declare that they have no conflicts of interest.

\section{Authors' Contributions}

Mamadou Ousmane Ndiath conceived, planned, designed, and supervised the study. Tsiriniaina Rakotondranaivo and Mihajarilala Rakotoniaina Tanjona collected, identified, and performed susceptibility mosquitoes test. Tsiriniaina Rakotondranaivo, Solohery Fanomezana Randriamanarivo, and Mihajarilala Rakotoniaina Tanjona performed laboratory work. Inès Vigan-Womas and Milijaona Randrianarivelojosia provided technical, logistical, and scientific support. Mamadou Ousmane Ndiath performed data analysis, interpreted the results, and drafted the manuscript. Milijaona Randrianarivelojosia, Inès Vigan-Womas, and Mamadou Ousmane Ndiath made the critical revision of the manuscript for important intellectual content. All authors read and approved the final manuscript.

\section{Acknowledgments}

We are grateful to Mrs. Ann Allen and Dr Astrid Knoblauch who reviewed previous versions of this manuscript. We also thank Dr Paul Howell and Dr Melissa Avery from CDCAtlanta for CSP antibodies, PCR primers, and protocols. We also thank the personnel of Unité d'Entomologie Médicale at Institut Pasteur de Madagascar and the Tsararano villagers for their cooperation throughout the study. This work was supported by the Department of International Affairs, Institut Pasteur International Network (G4 Group), awarded by Ousmane NDIATH.

\section{References}

[1] "World Malaria Report 2016. Document WHO/HTM/GMP/ 2016.2, Geneva, Switzerland, World Health Organization," http://www.who.int/malaria/publications/world-malaria-report-2016/en/, Accessed Dec 2016.

[2] T. Kesteman, S. A. Rafalimanantsoa, H. Razafimandimby et al., "Multiple causes of an unexpected malaria outbreak in a hightransmission area in Madagascar," Malaria Journal, vol. 15, no. $1,2016$.

[3] T. Kesteman, M. Randrianarivelojosia, C. Mattern et al., "Nationwide evaluation of malaria infections, morbidity, mortality, and coverage of malaria control interventions in Madagascar," Malaria Journal, vol. 13, no. 1, article 465, 2014.

[4] "Rapport DLP 2016. Enquête sur les indicateurs du paludisme à Madagascar," https://dhsprogram.com/pubs/pdf/MIS23/MIS23 .pdf, Accessed Dec 2016.

[5] H. Ranson and N. Lissenden, "Insecticide resistance in african anopheles mosquitoes: a worsening situation that needs urgent action to maintain malaria control," Trends in Parasitology, vol. 32, no. 3, pp. 187-196, 2016.

[6] D. Randriantsimaniry, "Vector control in the epidemics of the Madagascar highlands," Santé, vol. 5, no. 6, pp. 392-396, 1995.

[7] J. Hamon, J. Mouchet, G. Chauvet, and R. Lumaret, "Review of 14 years of malaria control in the french-speaking countries of tropical africa and in madagascar. considerations on the persistence of transmission and future prospects," Bulletin de la Société de Pathologie Exotique et de ses Filiales, vol. 56, pp. 933971, 1963.

[8] S. Mercier and J. B. Razafindrakoto, "Results of 3 years' campaigning of disinsectization of homes in Tanarive," Bulletin de la Société de Pathologie Exotique et de ses Filiales, vol. 46, pp. 463-473, 1953.

[9] M. Razanamparany, A. Bosman, N. Ravelomanana, F. Randriamiharisoa, and L. Razafimantsoa, "The malaria epidemic in Antananarivo observed from Pediatric Service "A" of the Befelatanana General Hospital.," Parassitologia, vol. 31, no. 1, pp. 89-99, 1989.

[10] M. S. Razanamparany, F. A. Randriamiharisoa, N. J. Razanamparany, and V. Ramialimanana, "The malaria epidemic in Antananarivo from 1983 to 1994 as seen through the Pediatric Service A in the Befelatanana General Hospital," Santé, vol. 5, no. 6, pp. 382-385, 1995.

[11] D. Fontenille and I. Rakotoarivony, "Reappearance of anopheles funestus as a malaria vector in the antananarivo region, Madagascar," Transactions of the Royal Society of Tropical Medicine and Hygiene, vol. 82, no. 4, pp. 644-645, 1988.

[12] J. P. Lepers, D. Fontenille, M. D. Andriamangatiana-Rason, P. Deloron, and P. Coulanges, "Ecological factors in the renewed outbreak of malaria in Madagascar," Bulletin de Société de Pathologie Exotique, vol. 83, pp. 330-341, 1990.

[13] L. Marrama, E. Rajaonarivelo, S. Laventure, and P. Rabarison, "Anopheles funestus and rice culture on the Plateau of Madagascar," Cahiers/Santé, vol. 5, no. 6, pp. 415-419, 1995.

[14] D. Fontenille and F. Simard, "Unravelling complexities in human malaria transmission dynamics in Africa through a comprehensive knowledge of vector populations," Comparative Immunology, Microbiology \& Infectious Diseases, vol. 27, no. 5, pp. 357-375, 2004.

[15] B. Greenwood and K. Koram, "Malaria control in Africa: Progress but still much to do," The Lancet, vol. 383, no. 9930, pp. 1703-1704, 2014.

[16] S. Sougoufara, S. M. Diédhiou, S. Doucouré et al., "Biting by Anopheles funestus in broad daylight after use of long-lasting insecticidal nets: A new challenge to malaria elimination," Malaria Journal, vol. 13, no. 1, article 125, 2014. 
[17] J.-F. Trape, A. Tall, N. Diagne et al., "Malaria morbidity and pyrethroid resistance after the introduction of insecticidetreated bednets and artemisinin-based combination therapies: a longitudinal study," The Lancet Infectious Diseases, vol. 11, no. 12, pp. 925-932, 2011.

[18] M. Zaim, A. Aitio, and N. Nakashima, "Safety of pyrethroidtreated mosquito nets," Medical and Veterinary Entomology, vol. 14, no. 1, pp. 1-5, 2000.

[19] J. Hemingway, H. Ranson, A. Magill et al., "Averting a malaria disaster: Will insecticide resistance derail malaria control?" The Lancet, vol. 387, no. 10029, pp. 1785-1788, 2016.

[20] H. Ranson, R. N'guessan, J. Lines, N. Moiroux, Z. Nkuni, and V. Corbel, "Pyrethroid resistance in African anopheline mosquitoes: what are the implications for malaria control?" Trends in Parasitology, vol. 27, no. 2, pp. 91-98, 2011.

[21] J. Ratovonjato, M. Randrianarivelojosia, M. E. Rakotondrainibe et al., "Entomological and parasitological impacts of indoor residual spraying with DDT, alphacypermethrin and deltamethrin in the western foothill area of Madagascar," Malaria Journal, vol. 13, no. 1, 2014.

[22] "Rapport DLP 2017. Plan stratégique national de lutte contre le paludisme à Madagascar 2018-2022," https://www.pmi.gov/ docs/default-source/default-document-library/malaria-operational-plans/fyl7/fy-2017-madagascar-malaria-operational-plan .pdf?sfvrsn=6f, Accessed Nov 2017.

[23] J.-D. Rakotoson, C. M. Fornadel, A. Belemvire et al., "Insecticide resistance status of three malaria vectors, Anopheles gambiae (s.l.), An. funestus and An. mascarensis, from the south, central and east coasts of Madagascar," Parasites \& Vectors, vol. 10, no. 1, 2017.

[24] "PMI Report 2016. President's Malaria Initiative Country Insecticide Susceptibility Summaries," August 2016, https://www .pmi.gov/docs/default-source/default-document-library/toolscurricula/pmi-insecticide-susceptibility-summary-august-2016 .pdf?sfvrsn=8, Accessed Nov 2016.

[25] M. T. Gillies and D. De Meillon, "The Anophelinae of Africa South of the Sahara," Publications of the South African Institute for Medical Research, vol. 54, p. 343, 1968.

[26] T. N. Nepomichene, L. Andrianaivolambo, S. Boyer, and C. Bourgouin, "Efficient method for establishing F1 progeny from wild populations of Anopheles mosquitoes," Malaria Journal, vol. 16, no. 1, article 21, 2017.

[27] "Test procedures for insecticide resistance monitoring in malaria vector mosquitoes," WHO/ CDS/CPC/MAL/D editor, 2016 2nd ed.Geneva, Switzerland, World Health Organization, http://apps.who.int/iris/handle/10665/250677, Accessed Nov 2016.

[28] S. Randriamaherijaona, H. J. Velonirina, and S. Boyer, "Susceptibility status of Anopheles arabiensis (Diptera: Culicidae) commonly used as biological materials for evaluations of malaria vector control tools in Madagascar," Malaria Journal, vol. 15, no. 1, article no. 338, 2016.

[29] W. G. Brogdon, J. C. McAllister, and J. Vulule, "Heme peroxidase activity measured in single mosquitoes identifies individuals expressing an elevated oxidase for insecticide resistance," Journal of the American Mosquito Control Association, vol. 13, no. 3, pp. 233-237, 1997.

[30] J. Hemingway and W. G. Brogdon, "Techniques to Detect Insecticide Resistance Mechanisms (Field and Laboratory Manual)," 1998.

[31] M. L. O. Sangba, T. Deketramete, S. P. Wango, M. Kazanji, M. Akogbeto, and M. O. Ndiath, "Insecticide resistance status of the
Anopheles funestus population in Central African Republic: A challenge in the war," Parasites \& Vectors, vol. 9, no. 1, 2016.

[32] M. L. Dustin, "Visualizing Immune System Complexity," Science Signaling, vol. 2, no. 66, pp. mr4-mr4, 2009.

[33] A. Cohuet, F. Simard, J.-C. Toto, P. Kengne, M. Coetzee, and D. Fontenille, "Species identification within the Anopheles funestus group of malaria vectors in Cameroon and evidence for a new species." The American Journal of Tropical Medicine and Hygiene, vol. 69, no. 2, pp. 200-205, 2003.

[34] N. Cuamba, J. C. Morgan, H. Irving, A. Steven, and C. S. Wondji, "High level of pyrethroid resistance in an Anopheles funestus population of the chokwe district in mozambique," PLoS ONE, vol. 5, no. 6, 2010.

[35] M. E. Rakotondraibe, G. Le Goff, E. Rajaonarivelo et al., "Insecticide sensitivity in malaria vectors in high altitude Madagascar after five years of vector control," Archives de l'Institut Pasteur de Madagascar, vol. 66, no. 1-2, pp. 32-35, 2000.

[36] J. Ratovonjato, G. Le Goff, E. Rajaonarivelo, E. M. Rakotondraibe, and V. Robert, "Recent observations on the sensitivity to pyrethroids and DDT of Anopheles arabiensis and Anopheles funestus in the central Highlands of Madagascar; preliminary results on the absence of the kdr mutation in An. arabiensis," Archives de l'Institut Pasteur de Madagascar, vol. 69, no. 1-2, pp. 63-69, 2003.

[37] "Les irrigations en 1925 dans la plaine de Marovoay," in Extrait du bulletin économique de Madagascar, 23e Année No. 1, Service de l'hydraulique et des Ameliorations Agricoles, 1926.

[38] J. D. Lines and N. S. Nassor, "DDT resistance in Anopheles gambiae declines with mosquito age," Medical and Veterinary Entomology, vol. 5, no. 3, pp. 261-265, 1991.

[39] C. Czeher, R. Labbo, I. Arzika, and J.-B. Duchemin, "Evidence of increasing Leu-Phe knockdown resistance mutation in Anopheles gambiae from Niger following a nationwide long-lasting insecticide-treated nets implementation," Malaria Journal, vol. 7, article no. 189, 2008.

[40] J. M. Vulule, R. F. Beach, F. K. Atieli, J. M. Roberts, D. L. Mount, and R. W. Mwangi, "Reduced susceptibility of Anopheles gambiae to permethrin associated with the use of permethrin-impregnated bednets and curtains in Kenya," Medical and Veterinary Entomology, vol. 8, no. 1, pp. 71-75, 1994.

[41] C. Mulamba, J. M. Riveron, S. S. Ibrahim et al., "Widespread pyrethroid and DDT resistance in the major malaria vector anopheles funestus in East Africa is driven by metabolic resistance mechanisms," PLoS ONE, vol. 9, no. 10, 2014.

[42] “Net Mapping Projet. Raport 2017," http://netmappingproject .allianceformalariaprevention.com/data?title_selective=All\&region_selective $=$ Abia\&year_selective $=$ All\&field_non_endemic_ value $=0$ Accessed Dec 2016 .

[43] R. Djouaka, H. Irving, Z. Tukur, and C. S. Wondji, "Exploring mechanisms of multiple insecticide resistance in a population of the malaria vector Anopheles funestus in Benin," PLoS ONE, vol. 6, no. 11, 2011.

[44] M. O. Ndiath, A. Cailleau, E. Orlandi-Pradines et al., "Emerging knock-down resistance in Anopheles arabiensis populations of Dakar, Senegal: First evidence of a high prevalence of kdr-e mutation in West African urban area," Malaria Journal, vol. 14, no. $1,2015$.

[45] M. O. Ndiath, K. Eiglmeier, M. L. Olé Sangba, I. Holm, M. Kazanji, and K. D. Vernick, "Composition and genetics of malaria vector populations in the Central African Republic," Malaria Journal, vol. 15, no. 1, article 387, 2016. 
[46] R. G. Kloke, E. Nhamahanga, R. H. Hunt, and M. Coetzee, "Vectorial status and insecticide resistance of Anopheles funestus from a sugar estate in southern Mozambique," Parasites \& Vectors, vol. 4, no. 1, article 16, 2011.

[47] J. M. Riveron, S. S. Ibrahim, E. Chanda et al., "The highly polymorphic CYP6M7 cytochrome $\mathrm{P} 450$ gene partners with the directionally selected CYP6P9a and CYP6P9b genes to expand the pyrethroid resistance front in the malaria vector Anopheles funestus in Africa," BMC Genomics, vol. 15, no. 1, article 817, 2014.

[48] J. M. Riveron, H. Irving, M. Ndula et al., "Directionally selected cytochrome P450 alleles are driving the spread of pyrethroid resistance in the major malaria vector Anopheles funestus," Proceedings of the National Acadamy of Sciences of the United States of America, vol. 110, no. 1, pp. 252-257, 2013. 


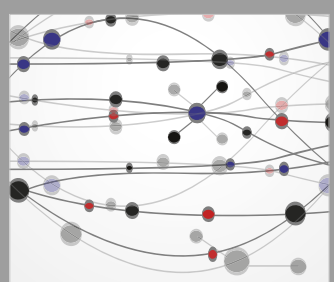

The Scientific World Journal
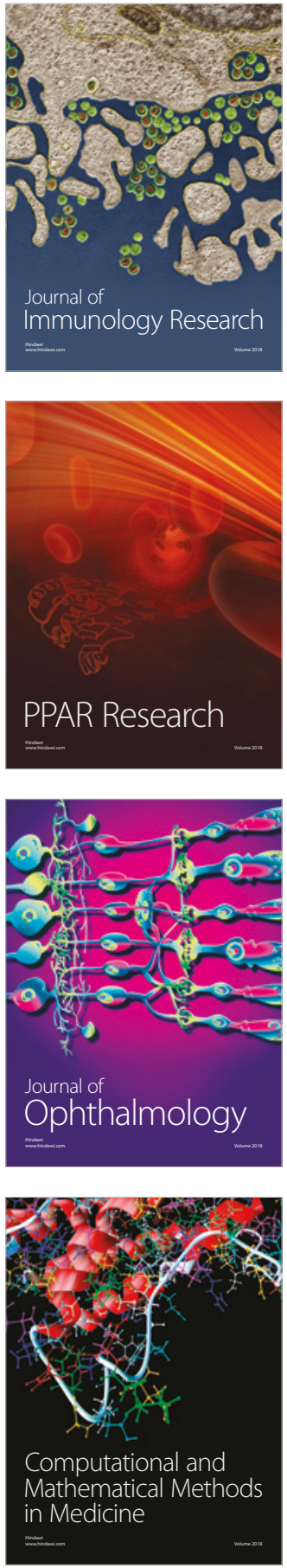

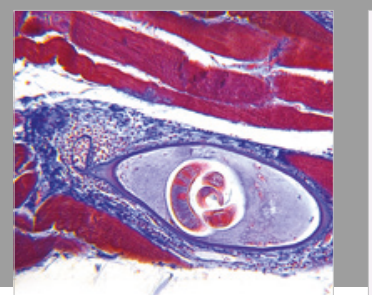

Gastroenterology Research and Practice

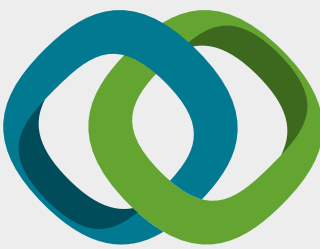

\section{Hindawi}

Submit your manuscripts at

www.hindawi.com
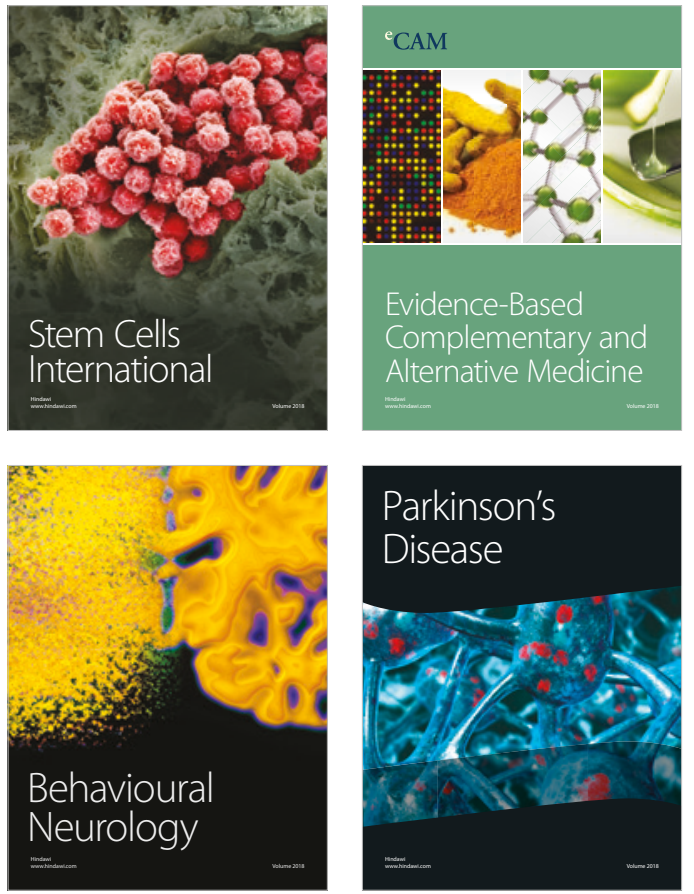

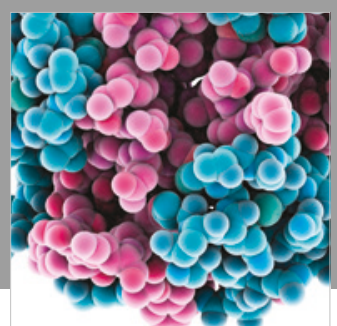

ournal of

Diabetes Research

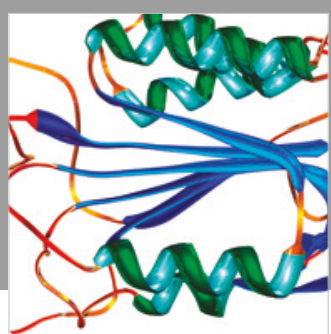

Disease Markers
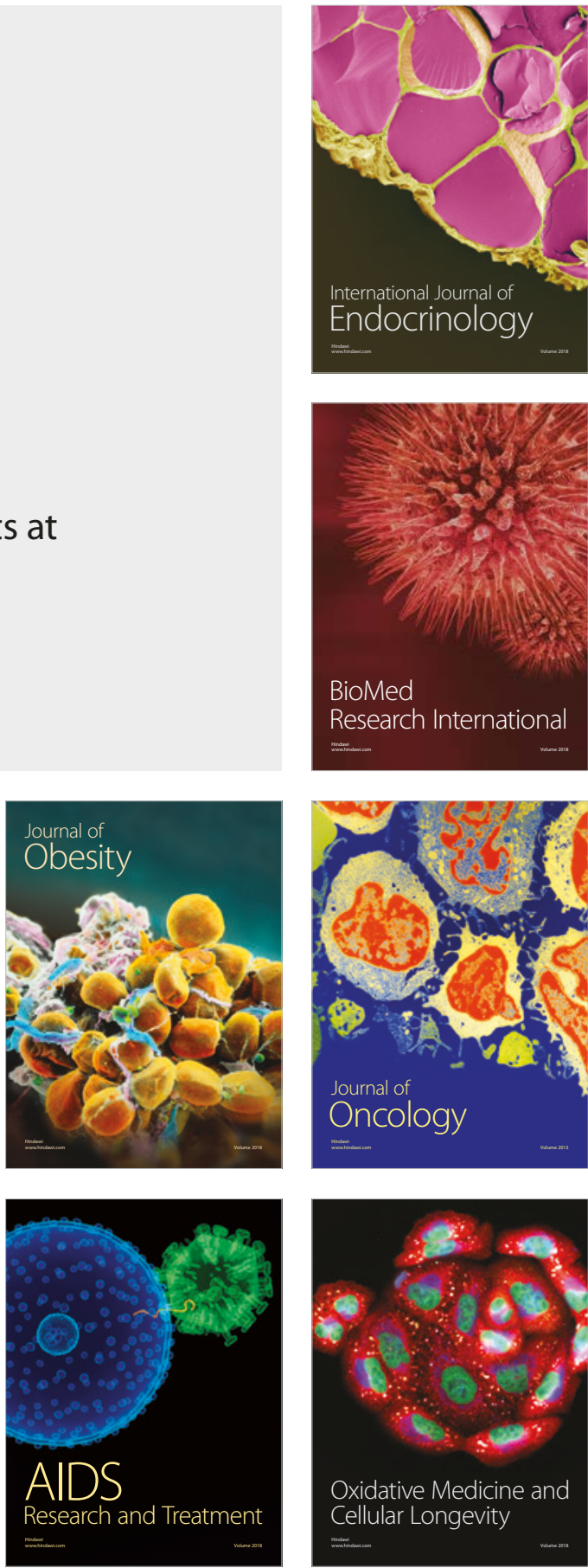